\title{
A New Contribution in Reducing Electric Field Distribution Within/Around Medium Voltage Underground Cable Terminations
}

\author{
S. S. Desouky \\ Electrical Engineering \\ Department \\ Port Said University \\ Port Said, Egypt \\ sobhyserry@yahoo.com
}

\author{
A. Z. El-Dein \\ Electrical Engineering \\ Department \\ Aswan University \\ Aswan, Egypt \\ azeinm2001@hotmail.com
}

\author{
R. A. Abd El-Aal \\ Electrical Engineering \\ Department \\ Port Said University \\ Port Said, Egypt \\ ramadanhv@gmail.com
}

\author{
N. A. A. El-Rahman \\ Electrical Engineering \\ Department \\ Workers University \\ Ismalia, Egypt \\ eng_nagwa77@yahoo.com
}

\begin{abstract}
In medium voltage cables, the stress control layers play an important part in controlling the electric field distribution around the medium voltage underground cable terminations. Underground cable accessories, used in medium voltage cable systems, need a stress control tube in order to maintain and control the insulation level which is designed for long life times. The term "electrical stress control" refers to the cable termination analysis of optimizing the electrical stress in the area of insulation shield cutback to reduce the electrical field concentration at this point in order to reduce breakdown in the cable insulation. This paper presents the effect of some materials of different relative permittivities and geometrical regulation with the curved shape stress relief cones on the electric field distribution of cable termination. The optimization was done by comparing the results of eight materials used. Also, the effect of the change in the thickness of the stress control tube is presented. The modeling design is very important for engineers to find the optimal solution of terminator design of medium voltage cables. This paper also describes the evolution of stress control systems and their benefits. A developed program using Finite Element Method (FEM) has calculated a numerical study to the stress control layering electric field distribution.
\end{abstract}

Keywords-stress control analysis; cable terminations; COMSOL analysis; stress control tube (SCT)

\section{INTRODUCTION}

Distribution of power is generally implemented using overhead lines or underground cables [1]. Underground cables are commonly used in low voltage and medium voltage solutions. The development of medium voltage underground cable accessories requires basic understanding of the stress caused by the electric field in different parts of the insulating structures. Material properties and product design play a key role in the durability of terminations and joints [2]. Terminations and joints are needed to make connections between lines or to an electrical device [3]. The main accessories of the underground power cables are terminations. Different sides are considered when designing the cable terminations because it must have the same quality as its associated cables if one wants to make connections between lines [3]. In cable composition, medium power cables need electrical stress control when terminated. When the insulation shield is removed from a cable, high potential values are concentrated at this point, presenting high electrical stress. Electric field increase at those points can cause local discharges that produce either flashover along the insulation surface or insulation failure causing cable breakdown. The design of underground cable terminations must eliminate the electric field concentration to reduce the breakdown of the cable. Or, the electrical field has to be controlled in a cable termination [3]. Underground cable accessories used in medium voltage cable systems need a highly reliable stress control system in order to maintain and control the insulation level which is designed for estimated life times longer than 30 years [4]. At the shield cut-back of a termination, an increase of the electric field strength happened, which makes discharges in the first time and later on to breakdown or flashover. Stress control materials are used to obtain a better field distribution in the cable termination [5]. A screening is the weakest area in a termination. Terminations without stress control suffered from the high electric field peak and at the end have failed with high voltage [6]. In electrical engineering, an insulated electrical conductor fitting into a grounded screen, is a common configuration that causes problems in the electric field distribution. Near the screen there is a critical area where an increase of the electric field happens [7].

\section{REQUIREMENTS OF STRESS CONTROL ON CABLE TERMINATIONS}

The cable setting up is needed for all types of underground cable terminations to present a better electrical and mechanical quality. The installation of the cable termination is presented in [3]. The removal of the shield of the insulation area changes the radially symmetric capacitor build of the cable. There becomes a high stress point at the shield terminus after the insulation area is terminated. And, high potential gradients are present between the insulation and the surrounding medium [8]. Medium voltage cable terminations should be controlled to 
reduce the stress that exists at a point where the screen or shield is terminated [9]. A precise determination of the electrical stress in insulated conductors will lead to an optimum design and a better selection of both wire size and the type of insulation for full utilization of the insulating conductors [10]. A graphical model of the cable termination can be found in [3]. There are five different areas in the model. These areas are: conductor $\left(\mathrm{R}_{1}\right)$, insulation $\left(\mathrm{R}_{2}\right)$, screen $\left(\mathrm{R}_{3}\right)$, stress control tube $\left(\mathrm{R}_{4}\right)$ and air $\left(\mathrm{R}_{0}\right)$.

\section{FINITE ELEMENT ANALYSIS}

The finite element analysis software needs to get suitable numerical formulation of the given nonlinear partial differential equation for the electric potential calculation. Hence, the original problem is replaced with its variation form of differential equation for the finite element model. The finite dimensional set is represented by a complete basis set and iterative solution is done to get accurate solution [11]. In highly non-homogeneous fields this assumption can lead to inaccurate results. Calculation accuracy can easily be improved by making the element net denser. This increases the amount of calculations and cannot be done unlimitedly [12]. COMSOL Multiphysics is a simulation software package that uses finite element method. The basic idea of FEM is to divide the examined area to small elements where each field's magnitude is described by a function. The potential $\mathrm{v}$ is used as field magnitude when static electric fields are examined minimizes interfaces from fluid flow and heat transfer to structural mechanics and electromagnetic analyses [13].

\section{FINITE ELEMENT COMPUTION}

Finite element computations were taken in 2-D dimensions with the ground screen modeled as floating with a dielectric of 1000. In the computation, the conductor screen was not modeled. The cable was typical of $10 \mathrm{kV}$ class cable with conductor of $13.6 \mathrm{~mm}$ diameter, insulation thickness $5.5 \mathrm{~mm}$ with dielectric constant 2.3 , the grounded screen was $0.7 \mathrm{~mm}$ thick with a dielectric constant 1000 . The dimensions of single core cable termination are provided in [14] and presented in table II. The algorithm for the finite element analysis Techniques starts with the Maxwell equations used in potential formulations. Then, the Laplace equations for the nonconducting regions and diffusion-like nonlinear equation for the stress control region are defined. Boundary Conditions combine these equations. These differential equations are solved by an iterative method to obtain the field distribution of the model [15]:

$$
\begin{array}{ll}
-\mathrm{P}_{0}[c(V) \mathrm{P} V]-f=0 & \\
c(V)= \begin{cases} & \forall k \in R_{0} \\
\varepsilon_{0} & \forall k \in R_{2} \\
\varepsilon_{0} \varepsilon_{i} & \forall k \in R_{4} \\
\sigma(\mathrm{E})+\frac{\varepsilon_{0} \varepsilon_{S C T}}{\Delta t} & \end{cases}
\end{array}
$$

$$
f=\left\{\begin{array}{ll}
0 & \forall k \in R_{0} \\
0 & \forall k \in R_{2} \\
-\varepsilon_{0} \varepsilon_{4}+\frac{\nabla^{2} V(t+\Delta t)}{\Delta t} & \forall k \in R_{4}
\end{array}\right\}
$$

where, $\mathrm{k}$ is a regular point in the defined region. The original problem is replaced with its variation form of differential equation for the finite element model. The finite dimensional set is represented by a complete basis set and iterative solution is done to get accurate solution. The solution method obtains nodal values of the variation form on each element. These potentials in vector form are then used in the system solution. Finally, the system of equations results in the form of:

$$
\mathrm{K} \cdot \mathrm{U}=\mathrm{F}
$$

where $\mathrm{K}$ is a $\mathrm{N} \times \mathrm{N}$ matrix, $\mathrm{U}$ is the nodal potential column vector, $\mathrm{F}$ is a $\mathrm{N}$ vector obtained by finite element method. [16]

\section{VALIDATION OF THE SIMULATION METHOD}

In this section the validation of the simulation results is done by comparing some simulation results with the experimental results obtained by others [1]. The effect of permittivity and conductivity were studied separately in[1]. The conductivities of the masses are generally between $10^{-8}$ and $10^{-}$ 12. The properties of tubes also vary greatly as relative permittivities from about 20 to 40 and conductivity from $10^{-7}$ to $10^{-13}[1]$. All simulations were done using the same boundary with $12 \mathrm{kV}$ and simulation setting by using COMSOL program. The results give that the use of a higher permittivity for the tube is better. It is seen that the permittivity affects the result more than conductivity does. The permittivity of the mass has a higher impact on the resulting peak. The highest permittivity for the mass and the tube results in about $25 \%$ lower electric field peak at the cutting point of the screening than the one that would be with the lowest permittivity values. The conductivity of materials does not affect the electric field peak greatly. The difference between the best and the worst setup is significant. The use of the highest permittivities and conductivities leads to about $55 \%$ lower electric field peak than without any grading. The lowest permittivities and conductivities lead to about 39\% lower electric field peak than without any grading [1]. Measuring the electric field can be so hard since the probe used for measurement can upset the field. The implementation of simulation results was carried out by studying the behavior of terminations in high voltage tests. The tests were done using a Baur PGK 110 / 5 HB high voltage test set. The broken terminations were cut open to study the reason of the failure [1]. Failures occurred at the cutting point of the screening which makes the insulation to melt and allowed current to flux between the conductor and the grounded cable screening. The hole in the insulation was not exactly at the cutting point but instead a bit away from it. This was considered the weakest point in the insulation. The cause of the failure is unknown since no clear installation error could be seen. The breakthrough could be a result of quality issues in the stress control mass or even in the cable insulation. Wrong 
electric properties in the mass could lead to a much higher electric field concentration at the cutting point of the screening. Improper structure of the mass could also give a breakthrough if air bubbles were left inside the termination [1]. Partial discharges generated inside the air bubbles would start to slowly burn the insulation and could lead to failure. The electric field peaks are comparatively lower than the $12 \mathrm{kV}$ voltages that were used in the simulations. It is however necessary to note that all simulations give perfect insulators without any air bubbles or uncleanness between layers. No insulator is ideal though and air bubbles exist in all areas. Therefore the resulting electric field distribution is always higher than that represented in simulations [1].

TABLE I. ELECTRIC FIELD PEAK RESULTS FOR TERMINATIONS [1].

\begin{tabular}{|c|c|c|}
\hline Simulation Setup & Permittivity $[\boldsymbol{\varepsilon} \mathbf{r}]$ & Field peak [kV/mm] \\
\hline $\mathbf{1}$ & 20 & 5.45 \\
\hline $\mathbf{2}$ & 40 & 5.45 \\
\hline $\mathbf{3}$ & 20 & 5.45 \\
\hline $\mathbf{4}$ & 40 & 5.45 \\
\hline $\mathbf{5}$ & 30 & 4.5 \\
\hline $\mathbf{6}$ & 30 & 4.5 \\
\hline $\mathbf{7}$ & 30 & 4.5 \\
\hline $\mathbf{8}$ & 30 & 4.5 \\
\hline
\end{tabular}

TABLE II. DIMENSION OF CABLE TERMINATION [14].

\begin{tabular}{|c|c|c|}
\hline Volt (kV) & $\begin{array}{c}\text { Indoor } \\
\text { termination }\end{array}$ & $\begin{array}{c}\text { outdoor } \\
\text { termination }\end{array}$ \\
\hline up to $12 / 20$ & 170 & 230 \\
\hline $18 / 30$ & 230 & 290 \\
\hline \multicolumn{2}{|c|}{ ( $\mathrm{z}$ ) dimension ( $\mathrm{mm})$} \\
\hline
\end{tabular}

\section{STRESS CONTROL TUBE (SCT) MODEL}

The application of stress control tube on the cable termination models is used considering the material properties. Thus, the thickness and the relative permittivity effects of the stress control area are presented using Finite Element Method. The favorable model design is done by using COMSOL program. In the cable termination model; the relative permittivity of the stress control tube is the variable properties. The effect of changes in the properties of the stress control tube is done by comparing the results of different simulations. The simulations solve the electric field distribution over the given model. The result analysis includes the electric field distribution at every point of the finite element model. So, the results are estimated as execution properties to optimize the design.

\section{RELATIVE PERMITTIVITY IMPACT ON THE STRESS CONTROL TUBE MODEL}

In our study, the effect of the relative permittivity of the stress control tube used on the underground cable termination is obtained. Stress control is also achieved by employing materials having relative permittivity significance higher than the cable dielectric. The relative permittivity of the stress control tube is a variable and is changed in each simulation.
The optimization was done by comparing the results obtained by 8 different materials. Stress control tube materials with relative permittivity changing from 5 to 1000 were used. The results of the analysis are obtained using COMSOL Multiphysics shown in following Figures with 2D and 3D Models of Electric field distribution. Figure 1 shows the maximum electric field distribution without SCT across the medium voltage cable termination. When, SCT is applied with $\varepsilon_{\mathrm{r}}=5$ (Figure 2) the electric field is decreased. Figure 6 presents the 3D Model of Electric field distribution with SCT $\varepsilon_{\mathrm{r}}=5$. Figure 4 shows that the peak value of electric field at cutting point is higher than when no stress control tube is used. The use of the highest permittivities leads to roughly 59\% lower electric field peak than without using SCT. The maximum electric field distribution with changing stress control tube relative permittivity and the values of maximum electric field at cutting point at the screen are shown in Table III. Figure 5 shows the maximum values of the electric field distribution with different relative permittivity. In Figure 6, the change of the relative permittivity is effective from 5 to 40 and the electric field does not change as the relative permittivity becomes greater than 40 , so the best effect of the stress control tube is with relative permittivity $\varepsilon_{\mathrm{r}}=40$. Figure 6 presents the Percentage of maximum values of the electric field reduction with different relative permittivity.

\section{THICKNESS EFFECT ON STRESS CONTROL TUBE MODEL}

The thickness of the stress control tube is changed from $1 \mathrm{~mm}$ to $5 \mathrm{~mm}$ and the relative permittivity is supposed to be constant with $\varepsilon_{\mathrm{r}}=40$. The results of the analysis are obtained by using COMSOL program. Figure 7 shows the Electric field distribution with $2 \mathrm{~mm}$ thickness. This value is compared with different thickness of the stress control tube in Table IV. From the obtained results presented with constant relative permittivity equal 40 , it is seen that the change of the stress control tube thickness has no effective role. For cost consideration, the best value of the cable termination model is considered with stress control tube of $1 \mathrm{~mm}$ thickness.

TABLE III. MAXIMUM VALUES OF THE ELECTRIC FIELD DISTRIBUTION WITH DIFFERENT RELATIVE PERMITTIVITY

\begin{tabular}{|c|c|}
\hline $\boldsymbol{\varepsilon} \mathbf{r}$ & $\begin{array}{c}\mathbf{E}_{\max } \\
(\mathbf{k V / m m})\end{array}$ \\
\hline 5 & 3.39 \\
\hline 10 & 2.731 \\
\hline 20 & 2.463 \\
\hline 40 & 2.463 \\
\hline 60 & 2.463 \\
\hline 100 & 2.463 \\
\hline 500 & 2.463 \\
\hline 1000 & 2.463 \\
\hline
\end{tabular}

TABLE IV. THE MAXIMUM VALUES OF THE ELECTRIC FIELD DISTRIBUTION FOR DIFFERENT THICKNESS OF THE STRESS CONTROL TUBE

\begin{tabular}{|c|c|c|c|c|c|}
\hline $\mathbf{d}(\mathbf{m m})$ & 1 & 2 & 3 & 4 & 5 \\
\hline $\begin{array}{c}\mathbf{E}_{\max } \\
\mathbf{( k V / m m})\end{array}$ & 2.473 & 2.463 & 2.474 & 2.458 & 2.459 \\
\hline
\end{tabular}




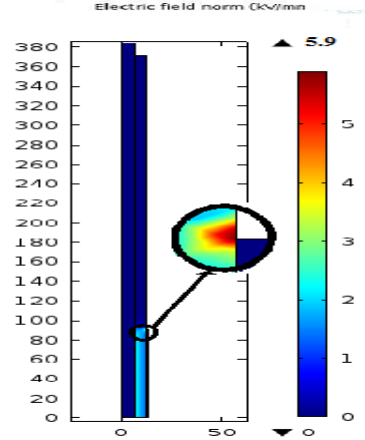

Fig. 1. Electric field distribution without SCT

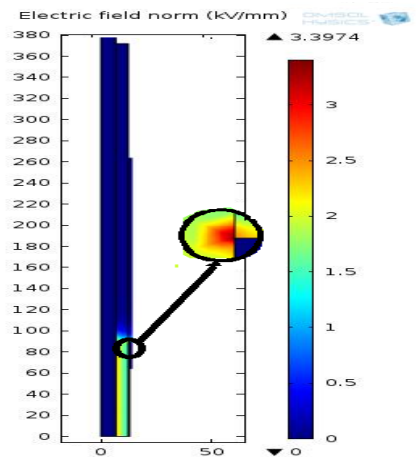

Fig. 2. Electric field distribution with SCT $\varepsilon_{\mathrm{r}}=5$

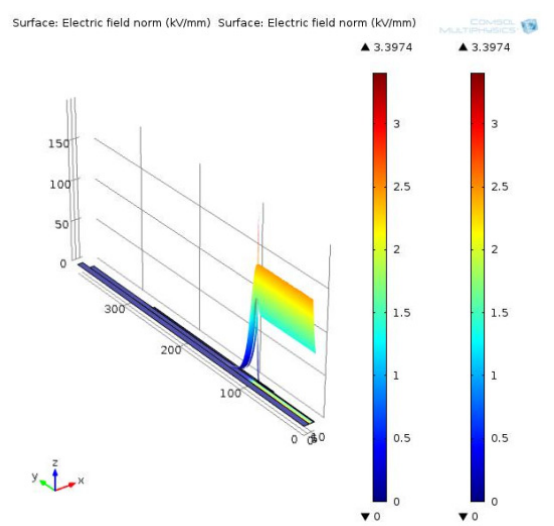

Fig. 3. The 3D Model of Electric field distribution with SCT $\mathrm{Er}=5$

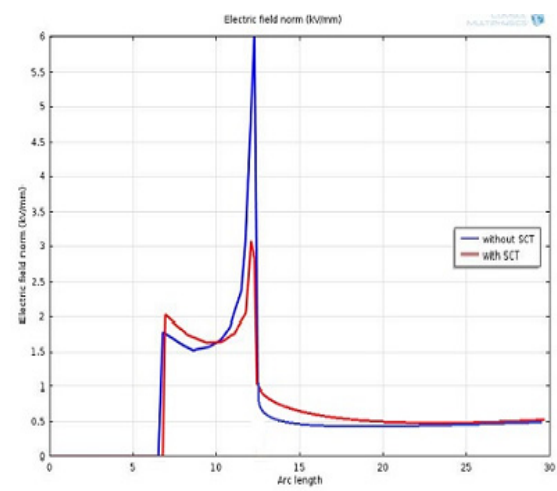

Fig. 4. The Maximum Electric Field distribution with and without SCT in the cable termination

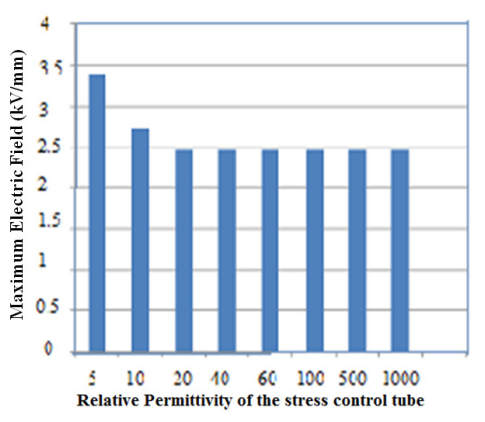

Fig. 5. Maximum values of the electric field distribution with different relative permittivity

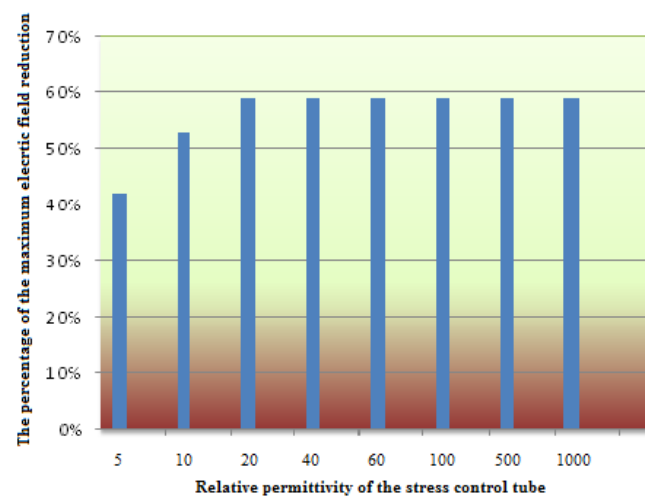

Fig. 6. The Percentage of maximum values of the electric field reduction with different relative permittivity

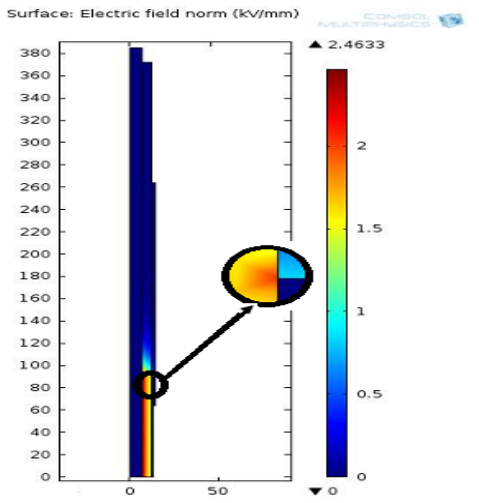

Fig. 7. Electric field distribution with $2 \mathrm{~mm}$ thick

\section{CABLE TERMINATION MODEL WITH DEFLECTOR}

The second method for terminating stress control is using the stress cones (geometric system). The stress cones use the geometrical structure for controlling. Geometric stress control involves an extension of the shielding extending the diameter at which the terminating cutout occurs and thereby reduces the stress at the cutout. The curved shape of the stress cones uniformly scatter the equipotential lines which outcomes reduced the electric field distribution the stress cone is extended beyond the screen termination, so that the potential gradient at the dielectric surface is reduced to a level where discharges will not occur. The numerical model of the deflector 
is presented with the COMSOL program. The results of the analysis are obtained as the electric field distribution using COMSOL Multiphysics with 2D and 3D models are shown in Figures 8 and 9. The deflector model reduced the concentrating of the electric field at the termination area with its geometrical structure. Figure 8 shows that the electric field is decreased with the curved geometry of the deflector model to $3.1 \mathrm{kV} / \mathrm{mm}$ compared with $5.9 \mathrm{kV} / \mathrm{mm}$ without stress control. Figure 9 shows the 3D Model of Electric field distribution with deflector. The deflector model should be achieved more neatly because it is determined as its ideal geometrical structure Ecological factors affect the effective implementation of the deflector. The implementation needs high versed working. So the total cost of the application is considered substantial disadvantage to implement the deflector.

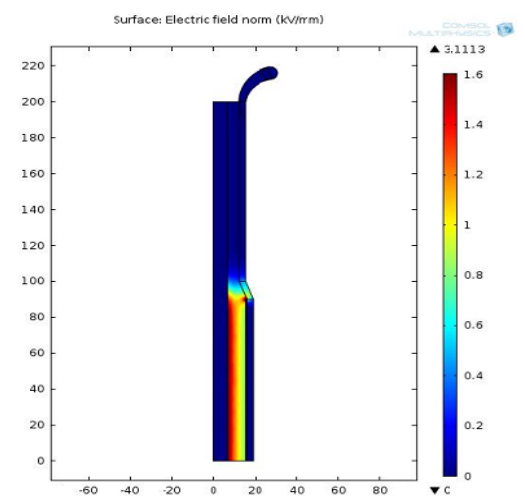

Fig. 8. Electric field distribution with deflector

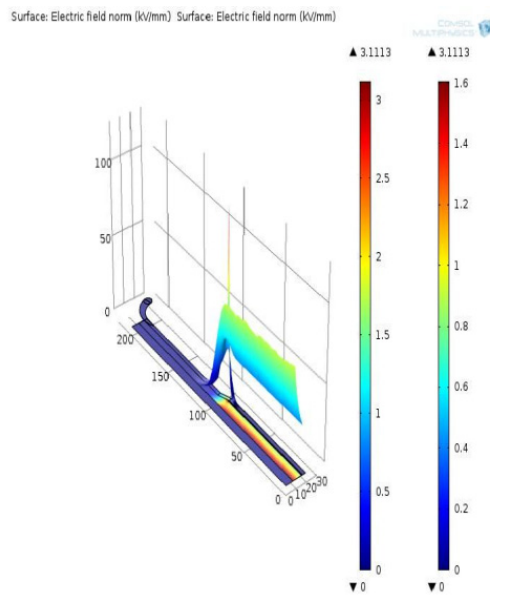

Fig. 9. The 3D Model of Electric field distribution with deflector

\section{COnClusions}

In this work, cable termination models with SCT and geometrical regulation with the stress relief cones are presented. The electric field distributions are simulated, and the results are evaluated. The stress control tube is consisting of a layer with different relative permittivity. The stress control tube applied on the cable screen reduces electric field at the termination area successfully. Increasing relative permittivity of SCT between 5 and 40 has effect. The stress effect is not seen in the screen cut point when the relative permittivity is increased greater than 40 . Hence, the effect of the electric field distribution is best achieved by a material with $\varepsilon_{\mathrm{r}}=40$. Also, from the obtained results it is clear that for relative permittivity 40, the change of the stress control layer thickness has no effect. For cost consideration, the best cable termination model is considered with stress control tube of $1 \mathrm{~mm}$ thickness. The deflector model decreases the concentrating electric field distribution at the termination area. The results show that the electric field is decreasing with the curved geometry of the deflector. The stress control tube reduces the electric field stress at the termination significantly compared with stress control cone.

\section{REFERENCES}

[1] K. Vakevainen, The effect of material properties to electric field distribution in medium voltage underground cable accessories, Degree Thesis, Arcada University, Finland, 2010

[2] M. Aro, J. Elovaara, M. Karttunen, K. Nousiainen, V. Palva, Suurjännitetekniikka, Otatieto, 2003

[3] G. Bas, Electric Field Analysis In Stress Controlled High Voltage Cables, MSc.Thesis, Middle East Technical University, 2005

[4] R. Strobl; W. Haverkamp; G. Malin; F. Fitzgerald, "Evolution of stress control systems medium voltage cable accessories", IEEE/PES Transmission and Distribution Conference and Exposition, Vol.2, pp. 843-848, 2001

[5] L. Bayon; F. Buret; C. Koelblin; T.Toledo, "Field distribution measurement and simulation of stress control materials for cable accessories", Proc. IEEE International Conference on Solid Dielectrics, Vol.2, pp. 534-537, 2004

[6] N. Raicevic; S. Ilic, S. Aleksic, R. Dimitrijevic, "Improving safety of cable networks by modeling deflectors of cable accessories," 4th International Conference on Power Engineering, Energy and Electrical Drives, Istanbul, pp. 70-75, 2013

[7] D. Carstea, I. Carstea, "Numerical simulation of electric field distribution in cable terminations", 6th International Conference on Telecommunications in Modern Satellite, Cable and Broadcasting Service, Vol. 2, pp. 479-482, 2003

[8] J. W. Hoffman, "Insulation enhancement with heat-shrinkable components", IEEE Electrical Insulation Magazine, Vol. 7, No. 2, pp. 33-38, 1991

[9] S. S. El-Dessouky, A. A. Rasmi, F. M. H. Youssef, "Experimental investigation of the flashover problem at the XLPE cable terminations under coastal environments," IEEE International Symposium on Electrical Insulation, Toronto, pp. 208-212, 1990

[10] A. A. Hossam-Eldin, S. S. Dessouky, S. M. El-Mekkawy, R. A. Abd ElAal, "Study and analysis of field enhancement and H.V rating of power cables containing micro cavities," Annual Report - Conference on Electrical Insulation and Dielectric Phenomena, Vancouver, pp. 797801,2007

[11] J. Jin, The Finite Element Method in Electromagnetics, IEEE Press

[12] COMSOL Multiphysics, AC/DC Module, available at: http://www. comsol.fi/products/acdc/

[13] A. Aarnio, Characterization of non-metallic materials for medium voltage cable accessories, MSc Thesis, Tampere: University of Technology, 2010

[14] Elastimold Egypt, Instalation Instruction Single Core Termination

[15] D. K. Cheng, Field and Wave Electromagnetics, Addison-Wesley Publishing Company

[16] C. Petrarca, D. Cerbasi, V. Tucci, M. Vitelli, "Numerical Analysis of Performances of Stress Grading Cable Accessories Made of Different Anisotropic Composite Materials", Annual Report Conference on Electrical Insulation and Dielectric Phenomena, Victoria, Vol. 2, pp. 494-497, 2000 